\begin{tabular}{|c|c|c|c|c|c|c|c|c|c|}
\hline I 901 & Hour & Mag. & Comparison stars & Weather & 1901 & Hour & Mag. & Comparison stars & Weather \\
\hline March & & & & & March & & & & \\
\hline 13 & $9^{\mathrm{h}}$ & 3.7 & $v$ Persei, $x$ Persei & Hazy & 27 & - & - & - & Cloudy \\
\hline 14 & 9 & 3.4 & $\varrho$ Persei, $\nu$ Persei & Partly cloudy & 28 & $9^{\mathrm{h}}$ & $5 \cdot 3$ & 1 and 30 Persei & Clear (moon) \\
\hline 15 & - & - & - & Cloudy & 29,30 & - & - & - & Cloudy \\
\hline 16 & $9^{\mathrm{h}}$ & 4.0 & $v$ Persei, $x$ Persei & Clear & 31 & $8^{\mathrm{h}}$ & 5.4 & l, $3^{\circ}, 3^{6}$ Persei & Full moon \\
\hline 17 & 8 & 3.8 & $\nu$ Persei & Clear & April & & & & \\
\hline 18 & 8 & 4.0 & $v$ Persei, $x$ Persei & Hazy & $i-6$ & - & - & - & Cloudy \\
\hline 19 & 8 & 5.0 & 1 Persei, 30 Persei & Hazy & 7 & $8^{\mathrm{h}}$ & 5.6 & $1,3^{\circ}, 3^{6}$ Persei & Clear \\
\hline 20 & - & - & - & Cloudy & 8 & - & - & - & Cloudy \\
\hline $2 \mathrm{I}$ & $8^{\mathrm{h}}$ & 5.2 & 1 and $3 \circ$ Persei & Clear & 9 & $9^{\mathrm{h}}$ & $5 \cdot 3$ & $1,3^{\circ}, 3^{6}$ Persei & Clear \\
\hline 22 & 8 & 4.8 & $\sigma$ and 1 Persei & Clear & Io & $8^{\mathrm{h}} 20^{\mathrm{m}}$ & 5.6 & 1, $30,3^{6}$ Persei & Clear \\
\hline 23 & - & - & - & Cloudy & II & $8^{\mathrm{h}}$ & 5.6 & 1, $3_{0}^{0}, 3^{6}$ Persei & Hazy \\
\hline 24 & $8^{\mathrm{h}}$ & 5.2 & $l$ and $3 \circ$ Persei & Clear & $12-15$ & - & - & - & Cloudy \\
\hline 25 & - & - & - & Rain & 16 & $8^{\mathrm{h}}$ & 5.6 & l, $3^{\circ}, 3^{6}$ Persei & Clear \\
\hline 26 & $8^{h}$ & 3.9 & $y$ and $x$ Persei & Clear & 17,18 & - & - & - & Cloudy \\
\hline
\end{tabular}

Remarks. Febr. 22. Bluish-white. - Febr. 25, Yellowish-white. - Febr. 27. Reddish. - March 2. Dull red. - March 13. Brilliant red. - March 26. Sudden rise. - March 28. Fall. - April ro. Yellowish-red.

University of Virginia, 1901 April 19.

\title{
Photographische Aufnahmen von Planeten
} a uf dem astrophysikalischen Observatorium Königstuhl.

\begin{tabular}{|c|c|c|c|c|c|c|c|c|c|c|c|c|}
\hline & Planet & 1901 & & M. Z & Heid. & & $\alpha$ & 8 & & Beob. - & Eph. & Beobachter \\
\hline$(391)$ & Ingeborg & Aug. & 8 & $10^{\mathrm{r}}$ & $43^{m} \cdot 3$ & & $16^{\mathrm{m}} \cdot 5$ & $+36^{\circ}$ & $39^{\prime}$ & $+3 \cdot 5$ & $-3^{8^{\prime}}$ & W \\
\hline (120) & Lachesis & Aug. & 8 & 13 & I I. 5 & $2 \mathrm{I}$ & I 3.8 & $-2 I$ & 5 & $+2 \cdot 3$ & +14 & $\mathrm{C}$ \\
\hline & & & 9 & I 3 & 38.0 & 21 & 12.8 & $-2 I$ & 7 & & & $\mathrm{C}$ \\
\hline (454) & Mathesis & Aug. & 8 & I 3 & I 1.5 & $2 \mathrm{I}$ & $54 \cdot 3$ & -22 & 55 & -0.1 & -1 & C \\
\hline$\left(3^{88)}\right.$ & Charybdis & Aug. & 8 & I 3 & I I. 5 & 2 I & 23.1 & -22 & 5 & +2.5 & +17 & $\mathrm{C}$ \\
\hline & & & 9 & 13 & $3^{8.0}$ & 21 & 22.3 & -22 & 7 & & & C \\
\hline$(104)$ & Klymene & Aug. & 9 & I 3 & 38.0 & $2 \mathrm{I}$ & I. 3 & -20 & 20 & -5.0 & +24 & $\mathrm{C}$ \\
\hline$(18)$ & Melpomene & Aug. & 9 & 10 & 48.0 & 22 & 20.9 & -8 & 14 & -2.0 & $-3^{2}$ & C \\
\hline & & & I 4 & I I & 18.5 & 22 & 18.1 & -9 & I 4 & & & W \\
\hline & & & 17 & I I & $3 \cdot 3$ & 22 & 16.1 & -9 & 53 & & & W \\
\hline (174) & Phaedra & Aug. & 9 & 10 & $4^{8.0}$ & 22 & 14.8 & $-I_{1}$ & 54 & -1.0 & +5 & C \\
\hline & & & 14 & I I & 18.5 & 22 & 10.3 & - II & 56 & & & $W$ \\
\hline (l $\left.\begin{array}{lll}1 & 2 & 2\end{array}\right)$ & Gerda & Aug. & 9 & 10 & 48.0 & 22 & 29.9 & -8 & 15 & -2.3 & +1 & $\mathrm{C}$ \\
\hline & & & 17 & I I & $3 \cdot 3$ & 22 & 24.5 & -8 & $\left.4^{8 *}\right)$ & & & W \\
\hline (2) & Pallas & Aug. & 17 & 10 & $27 \cdot 7$ & 23 & $7 \cdot 7$ & +5 & 32 & -1.6 & -28 & C \\
\hline & & & I 8 & 12 & 10.2 & 23 & 6.9 & +5 & 21 & & & C \\
\hline$(329)$ & Svea & Aug. & 17 & 10 & 27.7 & 22 & 59.5 & +3 & 24 & +0.7 & 0 & C \\
\hline & I $901 \mathrm{GQ}$ & Aug. & 17 & IO & $27 \cdot 7$ & 22 & $4 \mathrm{I} . \mathrm{J}$ & +6 & 25 & - & - & C \\
\hline & & & I 8 & I 2 & 10.2 & 22 & 40.2 & +6 & 26 & & & C \\
\hline
\end{tabular}

Viel Mühe haben uns (454) Mathesis, (391) Ingeborg und (315) Constantia gemacht. Vergeblich gesucht, wohl wegen der damaligen Lichtschwäche, hat Carnera am 13., 16. und 18. Juli nach (454) Mathesis (gefunden am 8. August). Nach (391) Ingeborg habe ich selbst vergebens gesucht am 8., 9. und I I. Jüli (gefunden am 8. August), nach (315) Constantia am 10., 14. und 17. August, sie wurde am 17. August mit (122) Gerda verwechselt und ist bis jetzt nicht gefunden. Ferner habe ich vergeblich gesucht nach (320) Katharina am 9. August und nach (251) Sophia am 10., I4. und 17. August.

Heidelberg, I go I Aug. 23.

Max Wolf.

*) Zunächst irrthümlich als Beobachtung des Planeten (315) Constantia angesehen und als solche schon in A. N. 3735 mitgetheilt.

Planet (48) Doris. Corr. der Ephem. Schilow (Bull. St. Pét. I 90 r Mai): Sept. $8+20^{\text {s }}$ - 1:7. Gr. $1^{\mathrm{m}}$. A. Abetti.

Planet (364) Isara. Corr. der Ephem. (Veröff. R. I. Nr. 15): Sept. $18+1^{\mathrm{m}} 5^{\mathrm{s}}+10: 6$. W. Luther. 